\title{
Cellular production of a counterfeit viral protein confers immunity to infection by a related virus
}

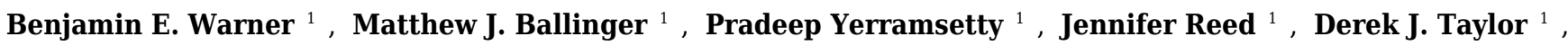 \\ Thomas J. Smith ${ }^{2}$, Jeremy A. Bruenn Corresp. 1 \\ 1 Department of Biological Sciences, The State University of New York at Buffalo, Buffalo, NY, USA \\ 2 Department of Biochemistry and Molecular Biology, University of Texas Medical Branch Galveston, Galveston, Texas, United States \\ Corresponding Author: Jeremy A. Bruenn \\ Email address: cambruen@buffalo.edu
}

DNA copies of many non-retroviral RNA virus genes or portions thereof (NIRVs) are present in the nuclear genomes of many eukaryotes. These have often been preserved for millions of years of evolution, suggesting that they play an important cellular function. One possible function is resistance to infection by related viruses. In some cases, this appears to occur through the piRNA system, but in others by way of counterfeit viral proteins encoded by NIRVs. In the fungi, NIRVs may be as long as 1400 uninterrupted codons. In one such case in the yeast Debaryomyces hansenii, one of these genes provides immunity to a related virus by virtue of expression of a counterfeit viral capsid protein, which interferes with assembly of viral capsids by negative complementation. The widespread occurrence of non-retroviral RNA virus genes in eukaryotes may reflect an underappreciated method of host resistance to infection. This work demonstrates for the first time that an endogenous host protein encoded by a gene that has been naturally acquired from a virus and fixed in a eukaryote can interfere with the replication of a related virus and do so by negative complementation. 
1

2

\section{Title Page}

4

5

6

7

23

24 virus

J.A. Bruenn

B.E. Warner

M.J. Ballinger

J. Reed

D.J. Taylor

T.J. Smith

Title: Cellular production of a counterfeit viral protein confers immunity to infection by a related

Running Title: Endogenous totivirus confers immunity

Authors: Benjamin E. Warner ${ }^{1}$, Matthew J. Ballinger ${ }^{1}$, Pradeep Yerramsetty ${ }^{1}$, Jennifer Reed ${ }^{1}$, Derek J. Taylor ${ }^{1}$, Thomas J. Smith ${ }^{2}$, and Jeremy A. Bruenn ${ }^{1 \#}$

${ }^{1}$ Department of Biological Sciences

State University of New York at Buffalo

Buffalo, NY 14260

${ }^{2}$ University of Texas Medical Branch at Galveston

Department of Biochemistry and Molecular Biology

301 University Boulevard

5.104D Basic Science Building, Route 0645

Galveston, Texas 77555-0645

${ }^{\#}$ Corresponding author

cambruen@buffalo.edu

benwarner@pitt.edu

mattball@uvic.ca

P. Yerramsetty

ypradeepkalyan@gmail.com

jennyree@buffalo.edu

djtaylor@buffalo.edu

thosmith@UTMB.EDU 


\section{Abstract}

48 DNA copies of many non-retroviral RNA virus genes or portions thereof (NIRVs) are present in

49 the nuclear genomes of many eukaryotes. These have often been preserved for millions of years

50 of evolution, suggesting that they play an important cellular function.

51 One possible function is resistance to infection by related viruses. In some cases, this appears to 52 occur through the piRNA system, but in others by way of counterfeit viral proteins encoded by 53 NIRVs. In the fungi, NIRVs may be as long as 1400 uninterrupted codons. In one such case in

54 the yeast Debaryomyces hansenii, one of these genes provides immunity to a related virus by 55 virtue of expression of a counterfeit viral capsid protein, which interferes with assembly of viral 56 capsids by negative complementation. The widespread occurrence of non-retroviral RNA virus 57 genes in eukaryotes may reflect an underappreciated method of host resistance to infection. This work demonstrates for the first time that an endogenous host protein encoded by a gene that has

59 been naturally acquired from a virus and fixed in a eukaryote can interfere with the replication of 60 a related virus and do so by negative complementation.

61

62

64 65 66 67 68 69

\section{Introduction}

The existence of genes derived from non-retroviral RNA viruses within the cellular genomes of eukaryotes (NIRVs) has been puzzling. Originally discovered in insects (Crochu et al. 2004), they have now been demonstrated in fungi (Frank \& Wolfe 2009; Liu et al. 2010; Taylor \& Bruenn 2009), plants (Chiba et al. 2011), mammals (Belyi et al. 2010; Horie et al. 2010; Taylor et al. 2011; Taylor et al. 2010) and other organisms (Katzourakis \& Gifford 2010;

8 Kondo et al. 2015). The origin of these sequences and their maintenance over millions of years remain to be explained. The origin of NIRVs is probably by adventitious integration via 
70 retrotransposon reverse transcription (Ballinger et al. 2012), but their maintenance is striking.

71 Although most NIRVs are pseudogenes, many preserve long open reading frames, which in the

72 fungi can be complete genomes reaching more than 1400 codons in length (Taylor \& Bruenn

73 2009). Some of these sequences have experienced purifying selection (Ballinger et al. 2013; Fort

74 et al. 2012; Taylor \& Bruenn 2009; Taylor et al. 2011). There is at least one case in which a

75 viral gene product (in this case a retroviral glycoprotein) has been appropriated for use by the

76 cell (Weiss \& Stoye 2013), but the widespread integration and persistence of viral capsid

77 polypeptide genes, RNA dependent RNA polymerase (RdRp) genes, and a variety of other viral

78 genes suggests the construction of a cellular armamentarium directed against RNA viruses.

One mechanism by which NIRVs might serve an antiviral function is through constitutive expression of siRNAs or piRNAs directed against related viruses(da Fonseca et al. 2016; Honda

81 \& Tomonaga 2016). This may be the case for flavivirus NIRVs in mosquitos (Palatini et al.

82 2017), but if this were the universal explanation for maintenance of NIRVs it would not explain

83 the maintenance of long open reading frames. In some of the fungi with NIRVs, there is no

84 RNAi system, since argonaut is missing (Drinnenberg et al. 2011; Drinnenberg et al. 2009).

85 Fujino et al. (2014) demonstrated that a bornavirus-like nucleoprotein element from ground

86 squirrels inhibited the replication of a related bornavirus in human cells (Fujino et al. 2014). The

87 ebolavirus vp35 NIRV protein from Myotis lucifigus appears to act as an interferon antagonist in

88 ebolavirus infection of human kidney cells (Kondoh et al. 2017). The only other known

89 expressed NIRV proteins are coded by totivirus-like genes in fungi (Taylor \& Bruenn 2009). We

90 chose one of these fungi, Debaryomyces hansenii (which lacks an RNAi system) to test the

91 antiviral function of NIRVs. 
94 polypeptide gene (cp1)(Taylor \& Bruenn 2009) and a single copy of a closely related capsid 95 polypeptide gene, cp2 (Taylor \& Bruenn 2009). D. hansenii belongs to the CTG clade of yeasts, utilizing an alternate genetic code, in which CUG codes for serine rather than leucine, but its

97 totivirus NIRVs are highly similar to the homologous genes in the Saccharomyces cerevisiae the function of these NIRVs is to repel invasion by the cognate totivirus, although no totivirus closely related to ScVL1 has been demonstrated in the CTG clade of yeasts. In fact, it has been postulated that genetic code alterations are a means of erecting a barrier against viral infection

102 (Holmes 2009). However, the totiviruses have breached this barrier, since Scheffersomyces segobiensis, another member of the CTG clade, is infected by a totivirus related to the $S$. cerevisiae virus ScVLa (ScVL-BC), which has managed to penetrate the codon barrier by losing almost all its CTG codons (Taylor et al. 2013). We sought to test one of the D. hansenii capsid polypeptide (cp) NIRVs (cp1) for its ability to cure infection with ScVL1, the virus to which it is

107

most closely related.

It has been known for some time that constitutive cellular expression of portions of the totivirus capsid polypeptides will interfere with the viral replication cycle and cure persistent totivirus infections in S. cerevisiae (Yao \& Bruenn 1995). The capsid of ScVL1, like that of the inner capsids of reoviruses and rotaviruses, is composed of 60 copies of an asymmetric dimer consisting of two copies of $\mathrm{cp}$ in two different conformations (Naitow et al. 2002a). Most of the dimer contacts occur in the first 435 amino acids of the ScVL1 capsid protein (cap), which is 680 amino acids long (Naitow et al. 2002a). ScVL1 cap N-terminal fragments containing at least 475 
115 amino acids are effective in curing ScVL1 (Yao \& Bruenn 1995), presumably acting by negative

116 complementation, successfully forming dimers but failing in subsequent viral assembly steps.

117 ScVLa cap N-terminal fragments are also effective in curing ScVLa. However expression of N-

118 terminal fragments of ScVL1 cap does not cure ScVLa or vice versa, which is not surprising

119 given that their caps are only $27 \%$ identical in sequence. The $D$. hansenii virus NIRV (DhV) cp1

120 is $42 \%$ identical to ScVL1 but only $21 \%$ identical to ScVLa, so we expected that it might cure

121 ScVL1 but not ScVLa.

122

123 Materials and Methods

124 Strains. Saccharomyces cerevisiae strains were from Paul Cullen. PC847 is MATa ura3-52

$125 \operatorname{trp} 1:: K A N$ [ScVL1, ScVLa, ScVM1]. Some isolates lack ScVLa. PC4391 is PPY640 MATa

126 ade2 his2 leu2 trp1 ura3 can1 FUS1::FUR1-lacZ::LEU2 [ScVL1, ScVLa, ScVM1].

127 Debaryomyces hansenii CBS767 was from Jean-Luc Souciet.

128

129 Plasmids. pYES2.1 (Thermo Fisher Scientific) is a GAL1 expression vector that provides C-

130 terminal VP5 and 6xHis-tags. pG3 is a GAPDH expression vector. Both are E. coli- S.

131 cerevisiae shuttle vectors selectable in yeast by URA3 (pYES) or by TRP1 (pG3) and in E. coli

132 by ampicillin resistance. Dhvcp1 DNA, optimized for expression in S. cerevisiae, was

133 synthesized by BioBasic and cloned into the BamHI site of pG3. It was subsequently subcloned

134 into pYES2.1 using topoisomerase cloning.

135 
136 RNA preparation. Crude RNA was prepared by whole cell phenol extraction (Bruenn \& Keitz

137 1976). For some experiments (Supplemental File 1), DNA was removed with RNase free DNase

138 (Promega) and the DNase denatured by heating.

139

140 RTPCR and qRTPCR. RTPCR was performed with the iTaq Universal Probes One-Step Kit

141 (BioRad) using the manufacturer's instructions and appropriate specific primers (see below) in a

142 BioRad T100 Thermal Cycler. Cycles were $50^{\circ} \mathrm{C}$ for 10 minutes, $95^{\circ} \mathrm{C}$ for 3 minutes; then 34

143 cycles of $95^{\circ} \mathrm{C}$ for 15 seconds and $60^{\circ} \mathrm{C}$ for 1 minute; finally $72^{\circ} \mathrm{C}$ for 5 minutes. qRTPCR was

144 performed with the $\mathrm{iTaq}^{\mathrm{TM}}$ Universal SYBR ${ }^{\circledR}$ Green Supermix (BioRad) in a BioRad C1000

145 Touch Thermal Cycler using the manufacturer's instructions with appropriate primers (Table 1).

146 Cycles were 2 minutes at $65^{\circ} \mathrm{C}, 30$ minutes at $50^{\circ} \mathrm{C}, 10$ minutes at $95^{\circ} \mathrm{C}, 30$ cycles of $(30$

147 seconds at $94^{\circ} \mathrm{C}, 30$ seconds at $50^{\circ} \mathrm{C}, 60$ seconds at $\left.72^{\circ} \mathrm{C}\right), 10$ minutes at $72^{\circ} \mathrm{C}$. We analyzed the

148 qPCR data using the $2^{\wedge}(-\Delta \Delta \mathrm{Ct})$ method (Livak \& Schmittgen 2001) and plotted the results as $149 \log _{10}$.

150

151 Protein purification. Proteins were prepared from whole cells with a proteinase inhibitor cocktail

152 (Sigma-Aldrich S8830) by French Press followed by low speed centrifugation (5 k x g) and 153 isolation of supernatant. His-tagged proteins were purified from the supernatant with nickel 154 magnetic beads (Biotool) according to the manufacturer's instructions and concentrated for 155 application to SDS-PAGE by acetone precipitation (Atallah et al. 2017). His-tagged proteins 156 were elaborated on 7.5\% SDS-PAGE (BioRad Mini-PROTEAN TGX gels). 
158 Western blotting. Proteins were transferred to PVDF filters (Merck-Millipore) using Mini-

159 PROTEAN Precast Gels (BioRad) using the manufacturer's instructions and apparatus.

160 Detection of his-tagged proteins was with mouse anti PENTA Histidine Tag:HRP (BioRad) and

161 SuperSignal West Dura extended duration substrate (Thermo Fisher Scientific) by enhanced

162 chemiluminescence and exposure to CL-XPosure film (Thermo Fisher Scientific). Protein

163 markers were BenchMark His-tagged protein standards (Thermo Fisher Scientific) and the

164 PageRuler Plus Prestained Protein Ladder (Thermo Fisher Scientific).

165 Mass spectroscopy. GeLC-MS/MS was performed on SDS PAGE fractions by the University of 166 Washington Proteomics Facility or by Bioproximity LLC.

167 Structure prediction. Modeling used the ESyPred3D Web Server 1.0 (Lambert et al. 2002) or the 168 I-TASSER server (Roy et al. 2010). Analysis of hybrid DhV-ScV capsids used the threaded 169 structure of the DhV capsid protein and the structure of the ScVL1(LA) virus (Naitow et al. 170 2002b). A portion of the virus capsid was generated using the VIPER database (Carrillo-Tripp et

171 al. 2009). Copies of the DhV subunits were then aligned to this LA scaffold using the program

172 COOT (Emsley \& Cowtan 2004). Using this assembly, the various interface surfaces were

173 analyzed using the online tool PDBePISA (Krissinel \& Henrick 2007).

174 PCR primers. Primers (IDT) used are described in Table 1

175 Note that the expected size for the RPS11B PCR fragment is derived from the mature mRNA,

176 minus the intron in the gene. The DhVcp1 sequence is not that of the original (GenBank

177 accession no. GQ291319.1) but is optimized for expression in S. cerevisiae with the same protein 178 sequence as the original (with the one CTG codon corrected to TCG). The ScVL1 sequence is 179 GenBank M28353.1. 


\section{Results}

DNAs coding for the DhV cp1 and ScVL1 cap were synthesized. The one CTG codon in

183 cp1 was altered to TCG to preserve the exact protein sequence present in D. hansenii and the 184 sequence optimized for codon expression in $S$. cerevisiae. The resultant sequence was cloned 185 into pG3 (Schena et al. 1991), a GAPDH expression vector and into pYES2.1, a GAL1 186 expression vector with several protein tags, including a 6xhis-tag, in the correct orientation for 187 protein expression. Cloning into pYES was performed so that the 6xhis-tag would follow the C188 terminus of cp1. Since a portion of the C-terminus of ScVL1 cap is unstructured in the assembled capsid (Naitow et al. 2002a) and since most of the contacts involved in capsid assembly are in the N-terminus, it was hoped that addition of a protein tag at the $\mathrm{C}$-terminus would not affect assembly. was transformed with pYEScp1 and $\mathrm{pG} 3 \mathrm{cp} 1$. When the transformants are grown in galactose minimal medium (PC847pYEScp1) or glucose minimal medium (PC847pG3cp1) for many generations, ScVL1 is cured (Fig. 1). The absence of ScVL1 is evident both by gel of ScVL1 (middle panel). Control RTPCR with primers from a ribosomal protein mRNA, bracketing an intron and therefore sensitive only to mature mRNA and not to genomic DNA, are positive with all RNA preparations (bottom panel). Interference with ScVL1 propagation is the result of protein interactions: transformation with similar expression vector constructs in which a 201 single base substitution ( $\mathrm{T}$ for $\mathrm{A}$ ) introduces a nonsense codon after codon 12 in the cp1 202 sequence (pG3cp1m and pYEScp1m) fails to cure ScVL1. That both the tagged (pYEScp1) and 
203 untagged (pG3cp1) vector constructs cure ScVL1 suggests that the tagged $\mathrm{cp} 1$ is equivalent to 204 the untagged version.

Despite the introduction of an early nonsense codon in cp1, the cp1 mRNA is still fully

206 expressed in the transformants (Supplemental file 1). Sample data from transformants of $S$. 207 cervisiae strain 4391, which has ScVL1, ScVM1, and ScVLa, are shown. In addition, the 208 specificity for interference with ScVL1 and not ScVLa is evident (Table 2 and Supplemental file 209 1). Table 2 shows results from a derivative of PC847 with both ScVL1 and ScVLa. This 210 demonstrates that the cp1 NIRV does cure its cognate virus and does so by expression of a 211 protein, not an RNA. The absence of ScVL1 viral particles in the cp1 transformants but their 212 persistence in the cplmut transformants was confirmed by tandem hybrid MS/MS of samples 213 from the 70-100 kDa region of a SDS-PAGE of extracted proteins (Table 2). This experiment 214 also demonstrates the curing of ScVL1 but not ScVLa by DhVcp1.

215

216

The explanation for this successful totivirus resistance probably resides in the structure of

217 cp1. We have shown previously that DhV cp1 and cp2 are incapable of forming viral particles 218 (Taylor \& Bruenn 2009). However, cp1 is predicted to be capable of mixed dimer formation 219 with ScVL1(LA) cap. The $42 \%$ sequence identity between the two proteins allows for a quite 220 adequate modeling of cp1 on the known structure of ScVL1(LA) cap (Naitow et al. 2002a).

221 Modeling using the ESyPred3D Web Server 1.0 (Lambert et al. 2002) on ScVL1(LA) cap gives 222 a predicted structure deviating from ScVL1(LA) cap by only 0.30 angstroms RMSD (excellent

223 agreement). Similarly, the I-TASSER server (Roy et al. 2010) gives a model with 0.46

224 angstroms RMSD. Analysis of hybrid DhV-ScV capsids used the threaded structure of the DhV 225 capsid protein and the structure of the ScVL1(LA) virus (Naitow et al. 2002b). A portion of the 
226 virus capsid encompassing the different contacts across icosahedral five-, three-, and two-fold

227 axes was generated using the VIPER database (Carrillo-Tripp et al. 2009). Copies of the DhV

228 subunits were then aligned to this LA scaffold using the program COOT (Emsley \& Cowtan

229 2004). Using this assembly, the various interface surfaces were analyzed using the online tool

230 PDBePISA (Krissinel \& Henrick 2007) and summarized in the table of Fig. 2. Assembly of

231 virions using these models shows that the subunit interaction interfaces for mixed DhV-ScV

232 subunits are all more stable than the DhV subunits by themselves but all are less stable than the

$233 \mathrm{ScV}-\mathrm{ScV}$ homo-subunit interfaces (Fig. 2). The interface generating the 3-fold axis of symmetry

234 especially is fatally flawed in the virions with DhV subunits. Consequently, DhVcp1 monomers

235 should participate in particle formation in the presence of ScVL1 cap and cause abortion of

236 particle formation by negative complementation.

237 This model for interference is corroborated by purification of capsid proteins from

238 PC847pYEScp1. Transformants of PC847 were selected on minimal glucose and then

239 transferred to galactose minimal medium for growth for several generations. If $\mathrm{cp} 1$ is capable of

240 forming multimers with ScVL1 cap, mixed multimers should be isolated by purification using $\mathrm{Ni}$

241 affinity chromatography of his-tagged $\mathrm{cp} 1$. Crude protein was isolated from PC847pYEScp1

242 grown in galactose or glucose minimal medium and PC847pYEScap grown in glucose minimal

243 medium. His-tagged proteins were purified using nickel magnetic beads and elaborated on $7.5 \%$

244 SDS-PAGE. Detection of his-tagged proteins was with an anti-his-tag HRP conjugated antibody

245 and a chemiluminescent substrate. Protein markers were his-tagged protein standards. Both the

246 pYEScp1 and pYEScap transformants produced purified his-tagged proteins of the expected size

247 (Fig. 3, lanes 3 and 4). However, only a minor fraction of cp1 in the crude protein preparation is

248 full-sized (lane 2); most of the cp1 is degraded. Because the his-tag is present on the C-terminus 
249 of the protein, only fragments retaining the C-terminus are detectable, but even so, fragments as

250 small as $10 \mathrm{kDa}$ are present. We suspect that degradation is the fate of $\mathrm{cp} 1$ monomers not

251 complexed with the viral capsid polypeptide (see Discussion). The control protein from

252 pYEScp1 transformants grown in glucose shows no $\mathrm{cp} 1$ at all, as expected (lane 1).

253 Several regions from an identical gel of purified his-tagged protein from a pYEScp1

254 PC847 transformant grown for a few generations in galactose were isolated and the included 255 proteins were analyzed by GeLC-MS/MS. The copurification of untagged ScV cap with the his256 tagged $\mathrm{DhV}$ cp1 implies the formation of mixed multimers. The cp1 his-tagged purified protein 257 is clearly present as a small proportion of cp1-cap multimers; there are about 16.5 cap monomers 258 for every cp1 monomer (Fig. 4). This is consistent with cp1 disrupting cap assembly by negative 259 complementation; the mixed multimers are the result of abortive capsid assembly of the viral 260 capsid from ScVL1 cap and the intrusion of cp1 his tagged monomers. The identification of cp1 261 and cap was made from numerous tryptic peptides (Supplemental file 2), essentially the same 262 peptides detected in previous experiments (Taylor et al. 2013) with the addition of one peptide 263 from the C-terminal tag of the $\mathrm{cp} 1$ his tagged protein. Note that although the two proteins are $26442 \%$ identical in sequence, they have no tryptic peptides in common, so they are easily 265 distinguished.

267 Discussion

268 Clearly, the cp1 NIRV has evolved from a capsid polypeptide capable of virion formation 269 to one that interferes with virion formation. Similar effects have been observed with endogenous 270 retrovirus and retrotransposon capsid genes (Mura et al. 2004; Tucker \& Garfinkel 2016). Since 271 all dsRNA viruses require an intact capsid for both transcription and replication, this provides a 
272 selective advantage to $D$. hansenii by freeing it of its totivirus burden. If totivirus NIRVs provide

273 an antiviral function and are under purifying selection (as evidenced by preservation of extensive

274 open reading frames), persistent totivirus infection must be disadvantageous. At least one

275 activity of totiviruses, stealing of caps from cellular mRNAs (Fujimura \& Esteban 2011) and

276 thereby making them untranslatable, seems likely to be unpalatable to the host. Significantly,

277 most of the predicted proteins of totivirus capsid polypeptide NIRVs have lost this activity

278 (Taylor \& Bruenn 2009).

279 Although the only totivirus presently known in the CTG clade is related to ScVLa(LBC)

280 rather than to ScVL1(LA) (Taylor et al. 2013), the persistence of NIRVs related to ScVL1 in $D$.

281 hansenii is not remarkable, given the similar persistence of filovirus NIRVs in mammals not

282 presently exposed to filoviruses (Taylor et al. 2010). Indeed, one filovirus NIRV does interfere

283 with filovirus infection in susceptible mammalian cells (Palatini et al. 2017). Since the $D$.

284 hansenii cp1 protein is rapidly degraded when not present in mixed multimers with ScVL1

285 capsid protein (Fig. 3), this may explain why it is not detectable in D. hansenii, even though its

286 mRNA is present (Taylor et al. 2013; Taylor \& Bruenn 2009). Presumably, the degraded

287 versions of cp1, even though some retain the his-tag, are not isolated by affinity chromatography

288 (Fig. 3) because they are folded in such a way that their carboxy termini are not accessible. They

289 are detected by Western blotting because they are completely unfolded in the process of transfer 290 to PVDF membranes.

291

292 Conclusion

293 We conclude that despite a very extensive evolutionary separation of S. cerevisiae and 294 D. hansenii, the latter preserves a functional NIRV conferring resistance to a virus in the former. 
295 Either we have captured a NIRV sequence prior to its disintegration in the absence of its cognate

296 virus or $D$. hansenii continues to retain a functional NIRV because it is periodically exposed to

297 this or a related totivirus by horizontal transfer.

298 At least in fungi, in which long open reading frames are preserved in NIRVs, their

299 function appears to be production of counterfeit viral proteins altered in such a way that their

300 interaction with elements of the cognate virus disrupts its replication cycle. Production of an

301 authentic NIRV from $D$. hansenii does cure $S$. cerevisiae of its cognate virus, and it does so by

302 negative complementation, rather than by interaction with cellular proteins (Kondoh et al. 2017).

303

304

305 Acknowledgements

306 We thank Yuko Ogata at the University of Washington and Bioproximity LLC for mass

307 spectroscopy, BioBasic Inc. for DNA synthesis, Laura Rusche for help with qRT-PCR

308 experiments, Gerald Koudelka for help in crude protein preparations, and Paul Cullen and Jean309 Luc Souciet for strains.

310

311 References

312 Atallah M, Flory MR, and Mallick P. 2017. A Robust Protocol for Protein Extraction and 313 Digestion. Methods Mol Biol 1550:1-10.

314 Ballinger MJ, Bruenn JA, Kotov AA, and Taylor DJ. 2013. Selectively maintained 315 paleoviruses in Holarctic water fleas reveal an ancient origin for phleboviruses. Virology 446:276-282. 
317 Ballinger MJ, Bruenn JA, and Taylor DJ. 2012. Phylogeny, integration and expression of

318

319

320

321

322

323

324

325

326

327

328

329

330

331

332

333

334

335

336

337

338

339

sigma virus-like genes in Drosophila. Molecular phylogenetics and evolution 65:251-258.

Belyi VA, Levine AJ, and Skalka AM. 2010. Unexpected inheritance: multiple integrations of ancient bornavirus and ebolavirus/marburgvirus sequences in vertebrate genomes. PLoS pathogens 6:e1001030.

Bruenn J, and Keitz B. 1976. The 5' ends of yeast killer factor RNAs are pppGp. Nucleic Acids Res 3:2427-2436.

Carrillo-Tripp M, Shepherd CM, Borelli IA, Venkataraman S, Lander G, Natarajan P, Johnson JE, Brooks I, C. L., and Reddy VS. 2009. VIPERdb2: an enhanced and web API enabled relational database for structural virology. . Nucleic Acids Res 37:D436-D442.

Chiba S, Kondo H, Tani A, Saisho D, Sakamoto W, Kanematsu S, and Suzuki N. 2011. Widespread endogenization of genome sequences of non-retroviral RNA viruses into plant genomes. PLoS pathogens 7:e1002146.

Crochu S, Cook S, Attoui H, Charrel RN, De Chesse R, Belhouchet M, Lemasson JJ, de Micco P, and de Lamballerie X. 2004. Sequences of flavivirus-related RNA viruses persist in DNA form integrated in the genome of Aedes spp. mosquitoes. J Gen Virol 85:1971-1980.

da Fonseca GC, de Oliveira LF, de Morais GL, Abdelnor RV, Nepomuceno AL, Waterhouse PM, Farinelli L, and Margis R. 2016. Unusual RNA plant virus integration in the soybean genome leads to the production of small RNAs. Plant Sci 246:62-69. 
340 Diamond ME, Dowhanick JJ, Nemeroff ME, Pietras DF, Tu C-L, and Bruenn JA. 1989.

$341 \quad$ Overlapping genes in a yeast dsRNA virus. $J$ Virol 63:3983-3990.

342 Drinnenberg IA, Fink GR, and Bartel DP. 2011. Compatibility with killer explains the rise of RNAi-deficient fungi. Science 333:1592.

344 Drinnenberg IA, Weinberg DE, Xie KT, Mower JP, Wolfe KH, Fink GR, and Bartel DP. 345 2009. RNAi in budding yeast. Science 326:544-550.

346 Emsley P, and Cowtan K. 2004. Coot: model-building tools for molecular graphics. Acta Cryst $D$ 60:2126-2132.

348 Fort P, Albertini A, Van-Hua A, Berthomieu A, Roche S, Delsuc F, Pasteur N, Capy P, Gaudin Y, and Weill M. 2012. Fossil rhabdoviral sequences integrated into arthropod genomes: ontogeny, evolution, and potential functionality. Molecular biology and evolution 29:381-390.

352 Frank AC, and Wolfe KH. 2009. Evolutionary capture of viral and plasmid DNA by yeast 353 nuclear chromosomes. Eukaryotic cell 8:1521-1531.

354 Fujimura T, and Esteban R. 2011. Cap-snatching mechanism in yeast L-A double355 stranded RNA virus. Proceedings of the National Academy of Sciences of the United States of America 108:17667-17671.

357 358

Fujimura T, and Wickner RB. 1988. Gene overlap results in a viral protein having an RNA binding domain and a major coat protein domain. Cell 55:663-671.

Fujino K, Horie M, Honda T, Merriman DK, and Tomonaga K. 2014. Inhibition of Borna disease virus replication by an endogenous bornavirus-like element in the ground squirrel genome. Proceedings of the National Academy of Sciences 111:1317513180. 
363 Holmes EC. 2009. The Emergence and Evolution of RNA viruses. Oxford: Oxford $364 \quad$ University Press.

365 Honda T, and Tomonaga K. 2016. Endogenous non-retroviral RNA virus elements 366 evidence a novel type of antiviral immunity. Mob Genet Elements 6:e1165785.

367 Horie M, Honda T, Suzuki Y, Kobayashi Y, Daito T, Oshida T, Ikuta K, Jern P, Gojobori 368 T, Coffin JM, and Tomonaga K. 2010. Endogenous non-retroviral RNA virus 369 elements in mammalian genomes. Nature 463:84-87.

Katzourakis A, and Gifford RJ. 2010. Endogenous viral elements in animal genomes.

371 PLoS genetics 6:e1001191.

372 Kondo H, Chiba S, and Suzuki N. 2015. Detection and analysis of non-retroviral RNA virus-like elements in plant, fungal, and insect genomes. Methods Mol Biol

374 1236:73-88.

375

376

377

378

379

380

381

382 383
Kondoh T, Manzoor R, Nao N, Maruyama J, Furuyama W, Miyamoto H, Shigeno A, Kuroda M, Matsuno K, Fujikura D, Kajihara M, Yoshida R, Igarashi M, and Takada A. 2017. Putative endogenous filovirus VP35-like protein potentially functions as an IFN antagonist but not a polymerase cofactor. PLoS One 12:e0186450.

Krissinel E, and Henrick K. 2007. Inference of macromolecular assemblies from crystalline state. J Mol Biol 372:774-797.

Lambert C, Leonard N, De Bolle X, and Depiereux E. 2002. ESyPred3D: Prediction of proteins 3D structures. Bioinformatics 18:1250-1256. 
384 Liu H, Fu Y, Jiang D, Li G, Xie J, Cheng J, Peng Y, Ghabrial SA, and Yi X. 2010.

385 Widespread horizontal gene transfer from double-stranded RNA viruses to 386 eukaryotic nuclear genomes. Journal of virology 84:11876-11887.

387 Livak KJ, and Schmittgen TD. 2001. Analysis of relative gene expression data using 388 real-time quantitative PCR and the 2(-Delta Delta $\mathrm{C}(\mathrm{T})$ ) Method. Methods 25:402-408.

390

Mura M, Murcia P, Caporale M, Spencer TE, Nagashima K, Rein A, and Palmarini M. 2004. Late viral interference induced by transdominant Gag of an endogenous retrovirus. Proc Natl Acad Sci U S A 101:11117-11122.

Naitow H, Tang J, Canady M, Wickner RB, and Johnson JE. 2002a. L-A virus at 3.4 A resolution reveals particle architecture and mRNA decapping mechanism. Nat Struct Biol 9:725-728.

Naitow H, Tang J, Canady M, Wickner RB, and Johnson JE. 2002b. L-A virus at 3.4 A 397 resolution reveals particle architecture and mRNA decapping mechanism. Nat Struct Biol 9:725-728.

399

400

401

402

403 404 405 406
Palatini U, Miesen P, Carballar-Lejarazu R, Ometto L, Rizzo E, Tu Z, van Rij RP, and Bonizzoni M. 2017. Comparative genomics shows that viral integrations are abundant and express piRNAs in the arboviral vectors Aedes aegypti and Aedes albopictus. BMC Genomics 18:512.

Roy A, Kucukural A, and Zhang Y. 2010. I-TASSER: a unified platform for automated protein structure and function prediction. Nature protocols 5:725-738.

Schena M, Picard D, and Yamamoto KR. 1991. Vectors for constitutive and inducible gene expression in yeast. Meth in Enzymol 194:389-398. 
407 Taylor DJ, Ballinger MJ, Bowman SM, and Bruenn JA. 2013. Virus-host co-evolution $408 \quad$ under a modified nuclear genetic code. PeerJ 1:e50.

409 Taylor DJ, and Bruenn J. 2009. The evolution of novel fungal genes from non-retroviral $410 \quad$ RNA viruses. BMC Biol 7:88.

411 Taylor DJ, Dittmar K, Ballinger MJ, and Bruenn JA. 2011. Evolutionary maintenance of 412 filovirus-like genes in bat genomes. BMC evolutionary biology 11:336.

413 Taylor DJ, Leach RW, and Bruenn J. 2010. Filoviruses are ancient and integrated into 414 mammalian genomes. BMC Evol Biol 10:193.

415 Tucker JM, and Garfinkel DJ. 2016. Ty1 escapes restriction by the self-encoded factor 416 p22 through mutations in capsid. Mob Genet Elements 6:e1154639.

417 Weiss RA, and Stoye JP. 2013. Virology. Our viral inheritance. Science 340:820-821.

418 Yao W, and Bruenn JA. 1995. Interference with replication of two double-stranded RNA viruses by production of N-terminal fragments of capsid polypeptides. Virology 214:215-

420 221.

421

422 Figures

423 Figure 1. Curing of ScVL1(ScVLA) by expression of DhVcp1.

424 Crude total RNA preparations from pYes transformants grown for many generations in 425 galactose minimal medium and from pG3 transformants grown for many generations in 426 glucose minimal medium were prepared by phenol extraction of whole cells. The top 427 panel is crude RNA run on a $1.4 \%$ agarose gel. The middle panel is RTPCR products 428 from these same samples using primers from the $R d R p$ region of $L 1$ on a $1.4 \%$ agarose 429 gel. The lower panel is RTPCR products from the same samples using primers from $S$. 
430 cerevisiae RPS11B, bracketing its intron, again run on a 1.4\% agarose gel. Markers

431 were the GeneRuler $1 \mathrm{~kb}$ DNA ladder (Thermo Fisher Scientific). This is a composite of

432 the three gels run with the same markers.

433

434

435 Figure 2. Predicted stability of DhVcp1-ScVcap interactions

436 Energy of formation of subunit surfaces. A. Ribbon diagram showing two-fold, three-

437 fold, and five-fold axes of symmetry. B. Subunit labels corresponding to the free

438 energies calculated in C. C. Free-energies of formation of subunit interaction

439 interfaces. Note that in the ScVL1(L-A) virus, there are two copies of the capsid protein

440 in the icosahedral asymmetric unit that are not in identical environments, labeled ' $A$ ' and

441 'B' in panel B. As shown in the figure, the A subunits are clustered around the 5 -fold

442 and 2-fold axes while the B subunits dominate the 3-fold axes.

443

444 Figure 3. Purification of $\mathrm{cp} 1$ his-tagged and cap his-tagged proteins

445 Western blotting of pYes derived his-tagged proteins and elaborated on a $7.5 \%$ SDS-

446 PAGE, transferred to PVDF membrane, probed with anti-his-tag antibody conjugated to

447 horse radish peroxidase (HRP), developed with an HRP substrate, and exposed to film.

448 Lane1 is crude protein from pYEScp1 transformed cells grown in glucose; lane 2 is

449 crude protein from pYEScp1 transformed cells grown in galactose; lane3 is pYEScp1

450 derived protein (from DhVcp1) purified using nickel affinity magnetic beads; and lane 4

451 is purified pYEScap1 derived protein (from ScVL1). Markers are his-tagged proteins 452 run in an adjacent lane. 
454 Figure 4. Detection of cp1 his-tagged - cap multimers

455 Relative intensities of pYEScp1 from DhV (DhV int.) and from ScVL1cap (ScV int) from 456 recovered tryptic peptides of gel fragments detected by GeLC-MS/MS from the purified 457 pYEScp1 protein of Fig. 3 from an identical gel run in parallel with the gel of Fig. 3.

458 Four fragments of the gel were analyzed, covering molecular weight ranges of 459 approximately $130-250 \mathrm{kDa}, 100-130 \mathrm{kDa}, 55-100 \mathrm{kDa}$, and 35-55 kDa. Both capsid 460 polypeptides were found only in the expected molecular weight range of full-sized 461 capsid polypeptides, with about 16.5 monomers of ScVcap to each purified his-tagged 462 DhVcp1. Intensities were relative molar amounts of the proteins calculated as averages 463 of the relative molar amounts of all detected tryptic peptides. 


\section{Figure 1}

\section{Curing of ScVL1(ScVLA) by expression of DhVcp1}

Crude total RNA preparations from pYes transformants grown for many generations in galactose minimal medium and from pG3 transformants grown for many generations in glucose minimal medium were prepared by phenol extraction of whole cells. The top panel is crude RNA run on a $1.4 \%$ agarose gel. The middle panel is RTPCR products from these same samples using primers from the RdRp region of L1 on a 1.4\% agarose gel. The lower panel is RTPCR products from the same samples using primers from S. cerevisiae RPS11B, bracketing its intron, again run on a 1.4\% agarose gel. Markers were the GeneRuler 1 kb DNA ladder (Thermo Fisher Scientific). This is a composite of the three gels run with the same markers. 
L1
dsRNA

L1 RdRp pYescp1 pYescp1m pG3cp1 pG3cp1m PC847 markers RTPCR

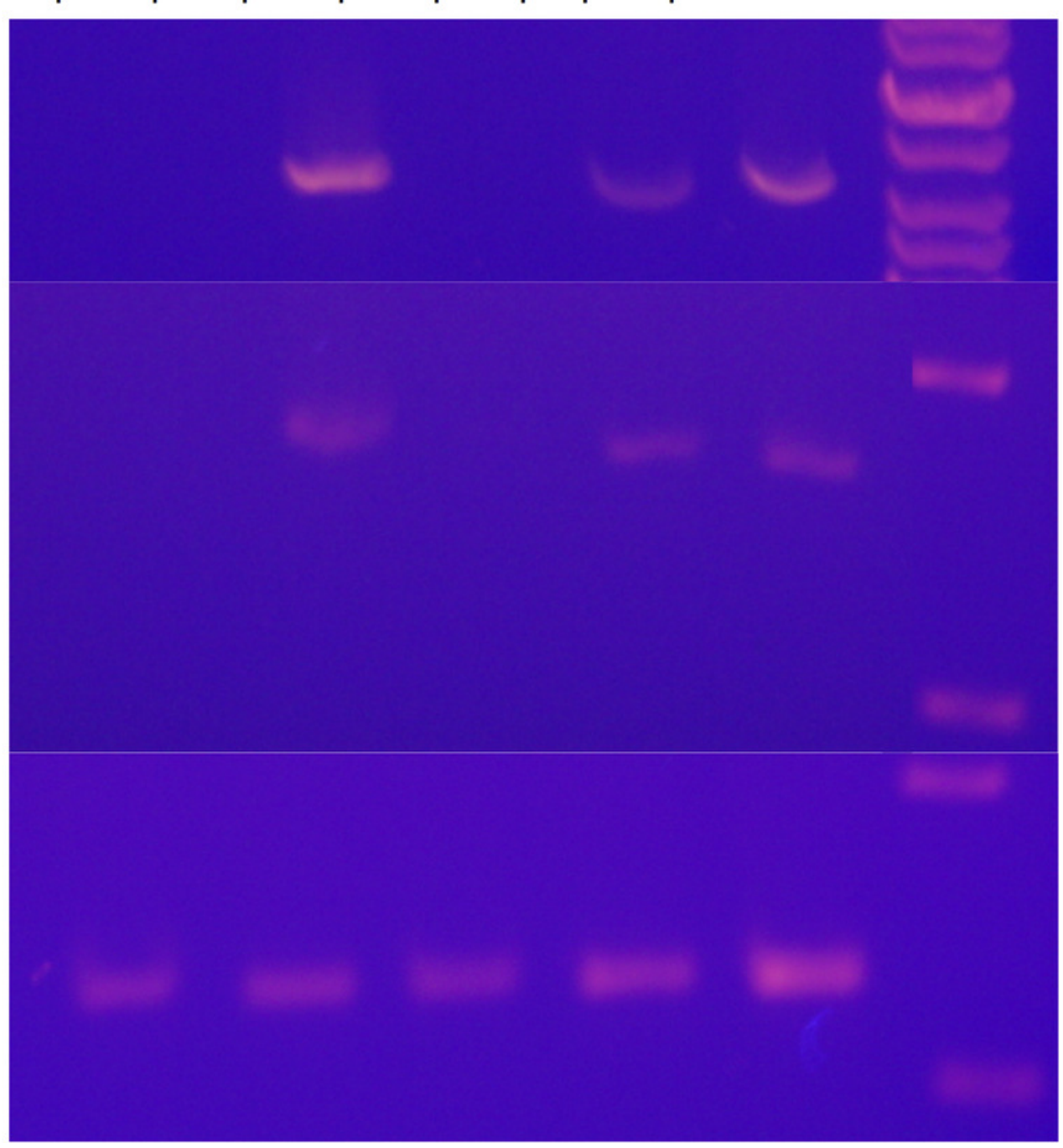

$8 \mathrm{kbp}$

$6 \mathrm{kbp}$

$5 \mathrm{kbp}$

$4 \mathrm{kbp}$

500bp

$361 b p$

$250 b p$

500bp

RPS11B

322bp

RTPCR

250bp 
Figure 2

\section{Predicted stability of DhVcp1-ScVcap interactions}

Energy of formation of subunit surfaces. A. Ribbon diagram showing two-fold, three-fold, and five-fold axes of symmetry. B. Subunit labels corresponding to the free energies calculated in C. C. Free-energies of formation of subunit interaction interfaces. Note that in the SCVL1(L-A) virus, there are two copies of the capsid protein in the icosahedral asymmetric unit that are not in identical environments, labeled ' $A$ ' and ' $B$ ' in panel $B$. As shown in the figure, the $A$ subunits are clustered around the 5 -fold and 2 -fold axes while the $B$ subunits dominate the 3 -fold axes.

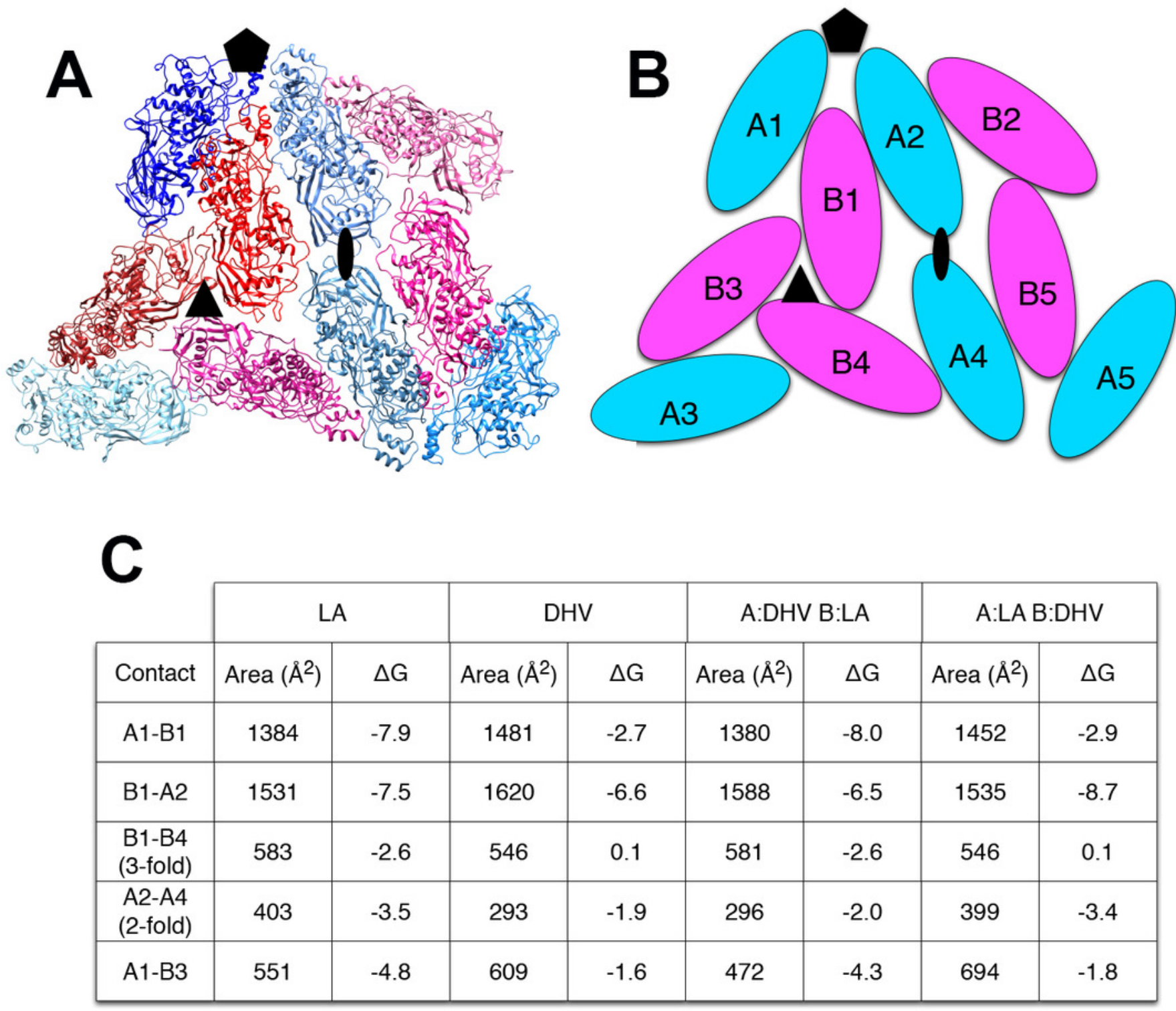




\section{Figure 3}

Purification of $\mathrm{cp} 1$ his-tagged and cap his-tagged proteins

Western of pYes derived his tagged proteins and elaborated on a 7.5\% SDS-PAGE,

transferred to PVDF membrane, probed with anti-his tag antibody conjugated to horse radish

peroxidase (HRP), developed with an HRP substrate, and exposed to film. Lane1 is crude

protein from pYEScp1 transformed cells grown in glucose; lane 2 is crude protein from

pYEScp1 transformed cells grown in galactose; lane3 is PYEScp1 derived protein (from

DhVcp1) purified using nickel affinity magnetic beads; and lane 4 is purified pYEScap1

derived protein (from ScVL1). Markers are his-tagged proteins run in an adjacent lane. 


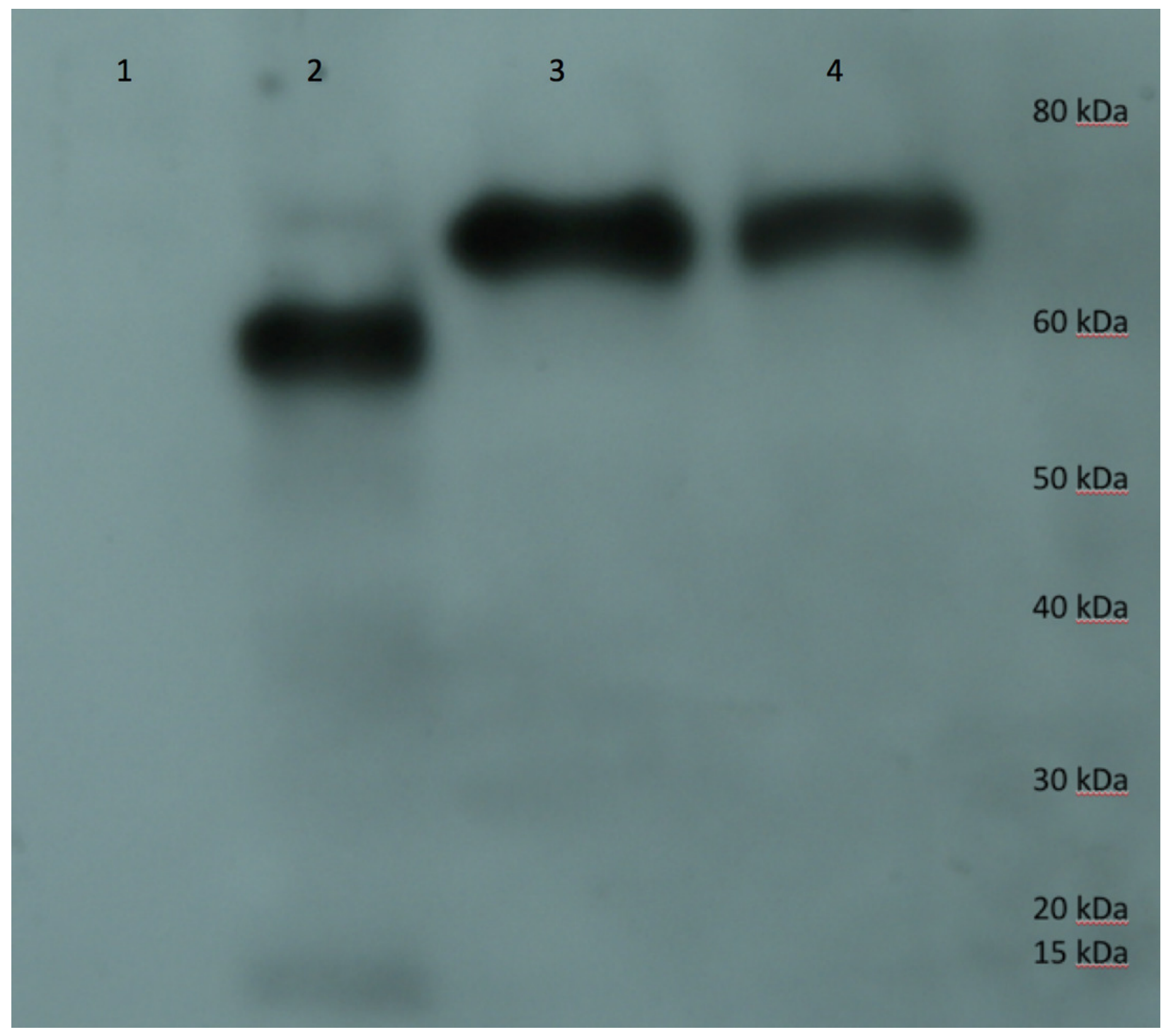




\section{Figure 4}

Detection of $\mathrm{cpl}$ his-tagged - cap multimers

Relative intensities of pYEScp1 from DhV (DhV int.) and from ScVL1cap (ScV int) from recovered tryptic peptides of gel fragments detected by GelLC-MS/MS from the purified pYEScp1 protein of Fig. 3 from an identical gel run in parallel with the gel of Fig. 3. Four fragments of the gel were analyzed, covering molecular weight ranges of approximately 130$250 \mathrm{kDa}, 100-130 \mathrm{kDa}, 55-100 \mathrm{kDa}$, and 35-55 kDa. Both capsid polypeptides were found in the expected molecular weight range, with about 16.5 monomers of ScVcap to each purified his tagged DhVcp1. Intensities were relative molar amounts of the proteins calculated as averages of the relative molar amounts of all detected tryptic peptides

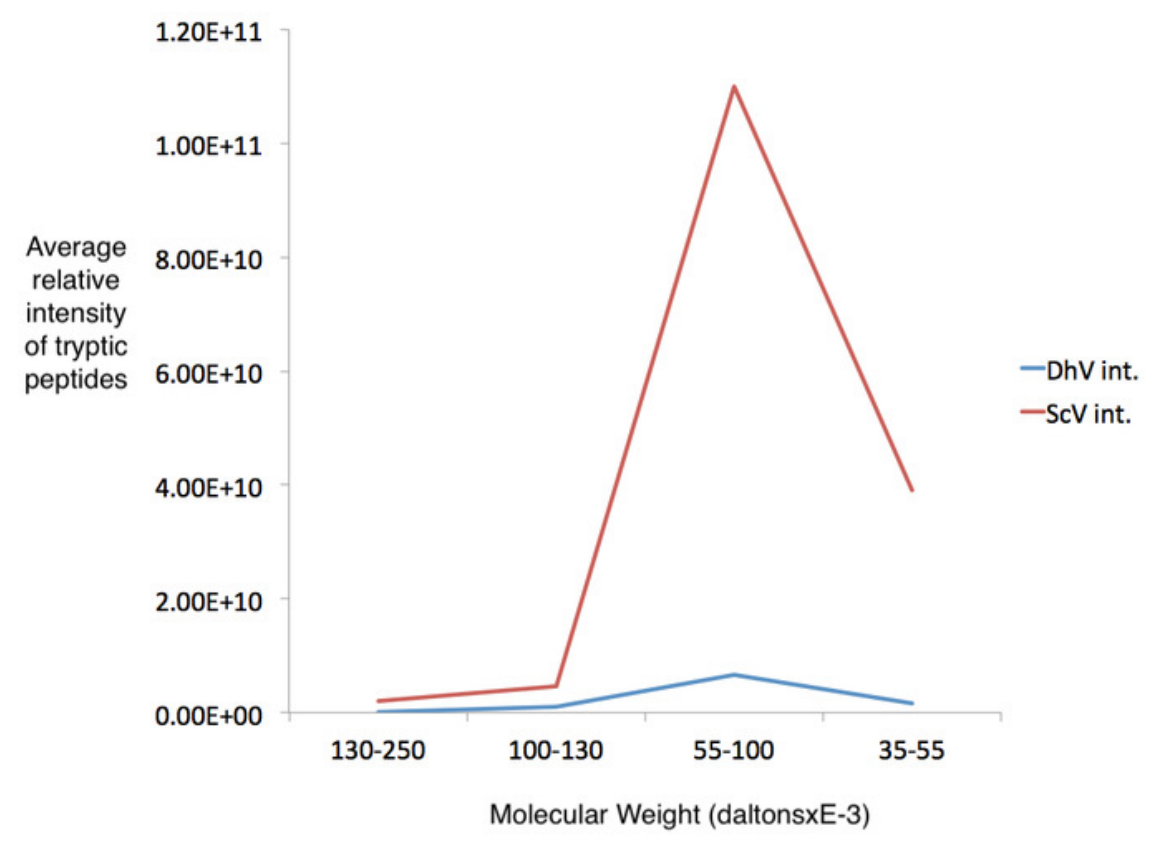




\section{Table $\mathbf{1}$ (on next page)}

Detection of ScV proteins by tandem hybrid MS/MS

Detection of ScV proteins in PC847 [ScVL1, ScVLa] transformed with pG3 vectors expressing DhV cpl or its derivative cpl mutant with a termination codon after the $12^{\text {th }}$ amino acid. The numbers of unique peptides detected for each protein by tandem hybrid MS/MS are indicated. 
1

\begin{tabular}{|l|l|l|l|l|}
\hline & Cp1 & Cp1 & Cp1 mutant & Cp1 mutant \\
\hline & Trypsin & Thermolysin & Trypsin & Thermolysin \\
\hline ScVL1 (L-A) & 0 & 0 & 14 & 17 \\
\hline ScVLa (L-BC) & 18 & 27 & 19 & 23 \\
\hline
\end{tabular}

2 Table 1. Detection of ScV proteins in PC847 [ScVL1, ScVLa] transformed with pG3

3 vectors expressing DhV cp1 or its derivative cp1 mutant with a termination codon after

4 the $12^{\text {th }}$ amino acid. The numbers of unique peptides detected for each protein by

5 tandem hybrid MS/MS are indicated.

6 
Table 2 (on next page)

Primers used for RTPCR 
1

\begin{tabular}{|l|l|l|l|l|}
\hline Primer & Gene & Sequence & Bases & Product (bp) \\
\hline L1rdrpf & ScVL1 RdRp & CGGCTATATTCGTGTGTGCG & $2945-2964$ & 361 \\
\hline L1rdrpr & ScVL1 RdRp & TGAGAACATCCTCGCACCTG & $3305-3286$ & 361 \\
\hline Lardrpf & ScVLa RdRp & AAGAGCACTACCTGACCGTG & $2731-2750$ & 337 \\
\hline Lardrpr & ScVLa RdRp & CTGCCTCCAGTACTCTTGCC & $3067-3048$ & 337 \\
\hline L1I & ScVL1 RdRp & TGGAAAAATTTCGGAGAACG & $4235-4254$ & 230 \\
\hline L1r & ScVL1 RdRp & ATGTTCGCCATTGGTGGTAT & $4464-4445$ & 230 \\
\hline Lacapl & ScVLa cap & GGCCTGTTATGAATCGAGGA & $1192-1211$ & 219 \\
\hline Lacapr & ScVLa cap & CAGTTTCCTGCCCCTCAATA & $1410-1391$ & 219 \\
\hline Cp1I & DhV cp1 & TTTGCCTCCGCAGTAAGTCT & $1216-1235$ & 206 \\
\hline Cp1r & DhV cp1 & TAAACCAAATACGGCGAACC & $1421-1402$ & 206 \\
\hline oLR0880 & Actin & GCCTTCTACGTTTCCATCCA & $391-411$ & 152 \\
\hline oLR0881 & Actin & GGCCAAATCGATTCTCAAA & $543-523$ & 152 \\
\hline RPS11Bf & RPS11B & CCACTGAATTAACTGTTCAATCTG & $5-28$ & 322 \\
\hline RPS11Br & RPS11B & ACTGGGACGTTCTTGTGTCT & $837-818$ & 322 \\
\hline
\end{tabular}

2

3 Table 2. Primers used for RTPCR.

4 